\title{
Lepidium s. str. (Brassicaceae) in the flora of Ukraine
}

\author{
Antonina P. Ilyinska
}

Department of Systematics and Floristics of Vascular Plants, M. G. Kholodny Institute of Botany, National Academy of Sciences of Ukraine, Tereshszenkivska 2, 01601 Kiev, Ukraine, e-mail: ilantonina@yandex.ru

\begin{abstract}
The key to the 14 species of Lepidium occurring in the Ukraine, including basionyms and the most important synonyms, and distribution of these species within the floristic regions, especially in Ukraine, are presented.
\end{abstract}

Key words: Lepidium, Brassicaceae, distribution, flora, Ukraine

\section{Introduction}

The Lepidium L. (Brassicaceae) genus comprises about 150-170 species widely distributed in temperate and subtropical regions, except the Far North and the Tropics, although it occurs in the mountains in the Tropics. The genus contains many taxonomically difficult species and infrageneric groups.

Globally, the Lepidium genus was investigated only once, by Thellung (1906) but there were numerous regional studies of the genus. For example, Smirnov (1948) studied species endemic to chalks and limestone areas in Eastern Europe, while Dorofeev (2012) reconsidered this genus for Eastern Europe in general; De Carvalho \& Vasconcellos (1964) studied all European species of the genus; Hewson (1982) dealt with Australian Lepidium.

K. Latowski (1982) applied anatomical characters of fruits and seeds in the taxonomy of Lepidium; P. Wąsowicz and A. Rostański ( 2009) analyzed quantitative characters of three frequently misdiagnosed annual species (L. virginicum, L. ruderale L., L. densiflorum Schrad.).

The Lepidium genus has been the subject of molecular studies for over 20 years. Molecular studies touched upon various taxa and focused on systematics (Mummenhoff et al. 2001), allopolyploidization events (Mummenhoff et al. 2004), evolution of flower structures (Bowman et al. 1999; Lee et al.2002), and development of dehiscent and indehiscent fruits (Mummenhoff et al. 2009) etc.

In Ukraine, the cruciferous family, including the Lepidium genus, was investigated by Kotov (1953) who recorded 10 species of Lepidium (excl. Cardaria Desv.) in "Flora of the URSR" (excluding the Crimea). Kotov made the then most detailed treatment of the Lepidium genus (and other cruciferous) in Ukrainian flora. In his later publications, Kotov $(1979,1987)$ listed 12 species (added two Crimean species, L. turczaninowii Lipsky and L. graminifolium L.) giving brief data concerning the distribution of all these species in Ukraine. Protopopova, Mosyakin and Shevera (1994) found in Transcarpathia and the Crimea new for the territory of Ukraine species - L. virginicum L. Later, in the LWKS herbarium, we found other specimens of this species, also collected in Transcarpathia: “Zakarpats'ka oblast, district Vynogradivs'ky, Eastern side of Koroleve settlement near railway and 'поблизу' quarry. 14.07.2006. Coll. O. Kagalo, O. Andreyeva, K. Doroshenko" (Ilyinska 2009). We presented L. pinnatifidum Ledeb. as another new species for Ukraine based on herbarium specimens (deposited in LE!) which was redefined by K. Latowski (Ilyinska 2005).

Leaf surface structure of four species of Lepidium and Cardaria were investigated by A. Ilyinska and M. Shevera (2004). Carrying out detailed taxonomic and floristic investigation of Lepidium in the flora of Ukraine, we selected lectotypes for two taxa: $L$. borysthenicum Kleopov and L. syvaschicum Kleopov (Ilyinska 2002). The results of taxonomic analysis of 13 species describing their morphological, biological and biomorphological characters, showing the current distribution of species in Ukraine (maps for each species applied) and reporting other features of species was presented in the fifth volume of "Ecoflora of Ukraine" (Ilyinska et al. 2007). 
The Lepidium genus is currently represented in Ukraine by 14 species. In this report, we summarize results of floristic investigation of Lepidium in the flora of Ukraine and provide the list of all species occurring in the country, including basionyms, the most important synonyms, nomenclatural types, and distribution of species within the floristic regions, especially in Ukraine, with the key to species.

\section{Material and methods}

The study was based on herbarium material deposited in CWB, DNZ, JALT, KW, LE, LW, LWKS, LWS, MW (abbreviations following Index Herbariorum: http://sweetgum.nybg.org/ih/), original research results and literature data.

\section{Results}

Key to Lepidium species in the flora of Ukraine.

1. Subshrubs. L. turczaninowii

- Perennials, biennials or annuals... .2

2. Fruits $4 \mathrm{~mm}$ or longer; valves broadly winged and papillate. L. campestre - Fruits less than $4 \mathrm{~mm}$; valves winged only above or not winged, glabrous or sparsely hirsute.

3. Stamens 2 or 4 . .3 Stamens 6 .....

4. Stamens 4. Petals shorter than sepals, 0.4-0.6 mm. Fruits not winged L. pinnatifidum - Stamens 2. Petals absent or rudimentary. Fruits apically winged.

5. Basal leaves 2- or 3-pinnatisect; upper cauline leaves linear, obtuse, entire L. ruderale - Basal leaves oblong, spatulate, or obovate, coarsely serrate, irregularly dentate, pinnatifid to lyrate; cauline leaves oblanceolate or linear, margins usually entire or irregularly serrate to dentate, rarely pinnatifid............6 6. Basal leaves rosulate; Petals absent or rudimentary, 0.3-0.9 mm, shortly than sepals. Fruits obovate to obovate-suborbicular, widest beyond the middle.

\section{L. densiflorum}

- Basal leaves not rosulate. Petals present (rarely rudimentary) $1.0-2.0 \mathrm{~mm}$, longer than sepals. Fruits orbicular or nearly so, widest in the middle.............. virginicum 7. Basal and lower cauline leaves 1- or 2-pinnatifid or pinnatisect.

- Basal and cauline leaves entire, not dissect............. 9

8. Cauline leaves pinnatisect or pinnatifid (middle) and linear, entire (upper). Petals white. Cotyledons 3-lobed, frequently. ..L. sativum - Cauline leaves (middle and upper) entire, ovate to cordate or suborbicular, base deeply cordate-amplexicaul. Petals pale yellow. Cotyledons entire, not dissect L. perfoliatum
9. Plants rhizomatous. Basal leaves not rosulate..........10

- Plants with caudex, not rhizomatous. Basal leaves rosulate ..11

10. Inflorescence dense, subcorymbose panicles; racemes slightly elongated or not in fruit.......L. latifolium - Inflorescence lax; racemes elongated in fruit. L. graminifolium

11. Basal leaves narrow, lanceolate. .12

- Basal leaves wide, ovate or suborbicular..............13 12.Plants $10-25 \mathrm{~cm}$. Cauline leaves shortly petiolate or sessile (upper), without auricles. Fruits broadly rhomboid, base abrupt-rounded. ..L. syvaschicum - Plants 6-13 cm. Cauline leaves sessile, subamplexicaule, with small acute auricles. Fruits ovate, acute-like, base truncate or subcordate. L. pumilum 13. Fruits 3-4 mm, ovate, acute to base and to apex. Basal leaves ovate; cauline leaves sagittate and amplexicaule L. crassifolium

- Fruits 2.5-3.0(3.5) mm, ovate-triangular, base obtuse or truncate. Basal leaves elliptic to orbicular; cauline leaves short-petiolate or sessile (upper), auricles present, somewhat obtuse L. borysthenicum

Synopsis of the Lepidium genus in the flora of Ukraine. Lepidium L. 1753, Sp. Pl.: 643. Lectotypus: L. latifolium L.

1. L. graminifolium L. 1759, Syst. Nat. ed. 10, 2: 1127; Kotov, 1979, Fl. evrop. ch. SSSR, 4: 60; Ilyinska et al. 2007, Ecofl. Ukr. 5: 80. - L. graminifolium subsp. graminifolium: Vasconcellos, Akeroyd, Rich, 1993, Fl. Europ. 1: 401. - L. gramineum Lam. 1778, Fl. Franç. 2: 469. - Thlaspi graminifolium (L.) Poiret. 1806, Encycl. Meth. Bot. 7: 546.

Described from Southern Europe: "Habitat in Europe australi [Sp. Pl. 1763, ed. 2, 2: 900]". Lectotypus: Herb. Linn. No. 824.15 (LINN) [Hedge, 2002, in Cafferty et Jarvis (ed.), Taxon, 51: 534].

Distribution in Ukraine: Crimea (Southern Coast). General distribution: southern part of Europe; Southwest Asia; Northwest Africa.

2. L. crassifolium Waldst. et Kit. 1799, Descr. Icon. Pl. Rar. Hung. 1: 4, tab. 4; Ilyinska et al. 2007, Ecofl. Ukr. 5: 80; V. I. Dorofeev, 2012, Konspekt Fl. Vost. Evr. 1: 424. - L. cartilagineum (J. Mayer) Thell. subsp. crassifolium (Waldst. et Kit.) Thell. 1906, Neue Denkschr. Schweiz. Ges. Naturw. 41, 1: 153; Vasconcellos, Akeroyd, Rich, 1964, Fl. Europ. 1: 332. $-L$. cartilagineum (J. Mayer) Thell. subsp. cartilagineum: Vasconcellos, Akeroyd, Rich, 1993, Fl. Europ. 1: 401, p. p. - L. cartilagineum auct. non (J. Mayer) Thell.: Kotov, 1953, Fl. Ukr. SSR, 5: 398; Kotov, 1979, Fl. evrop. ch. SSSR, 4: 59. 
Described from the Central Europe (Hungary): "Crescit in locis salsis siccis sterilissimis Comitatus Albensis solo instar lapidis duro in quo praeter novam Camphorosmas speciem fere nullum aliud vegetabile crescit.".

Distribution in Ukraine: Western Forest-steppe. General distribution: Central and East (south) Europe.

3. L. borysthenicum Kleopov, 1939, Journ. Inst. Bot. Akad. Nauk URSR, 21-22: 251; Kotov, 1953, Fl. Ukr. SSR, 5: 402; Vasconcellos, Akeroyd, Rich, 1993, Fl. Europ. 1: 401, p. p. - L. crassifolium auct. fl. Ukr. non Waldst. et Kit. p. p.: Schmalh. 1895, Fl. Mit., S. Rus., Crymea, N. Cauc. 1: 98; Kotov, 1979, Fl. europ. czasti USSR, 4: 59, p. p.

Described from the East Europe (Ukraine, Poltava area). Lectotypus: "Thlaspi rotundifolium Gaud. Poltavskaja gubernia, Lubny. Na solonczakakh, okolo Beresotoczi. 1849, Awgustinovicz" (Полтавская губерния, Лубны. На солончаках, около Березоточи. 1849 г. Августинович) (our italics; the surname was written by M. Kotov) (KW) [Ilyinska 2002, Ukr. Botan. Journ. 59, 2: 261].

Distribution in Ukraine: Forest-steppe, Steppe, Crimea. General distribution: East Europe (south).

4. L. pumilum Boiss. et Balansa, 1859, in Boiss. Diagn. Pl. Orient. ser. 2, 6: 21; Kotov, 1979, Fl. evrop. ch. SSSR, 4: 60; Ilyinska et al. 2007, Ecofl. Ukr. 5: 80. - L. crassifolium Waldst. et Kit. var. pumilum (Boiss. et Balansa) Thell. 1906, Mitt. Bot. Mus. Zurich, 28: 153, 155; N. Busch, 1907, Fl. Cauc. Crit. 3, 4: 111.L. cartilagineum subsp. pumilum (Boiss. et Balansa) Hedge, 1968, in Rechinger, Fl. Iran. 57: 67; Vasconcellos, Akeroyd, Rich, 1993, Fl. Europ. 1: 401, p. p.

Described from M. Asia (Anatolia): "Hab. in salsis ad margines paludum ad occidentem Caesareae sitarum alt. 1100 metr. cl. Balansa qui legit fructiferam Sept. 1856". Lectotypus: "In salsis ad margines paludum ad occidentem Caesareae, Balansa" (G) [Hedge (1968) in Dorofeev, 2008, Botan. Journ. 93, 12: 1967].

Distribution in Ukraine: Steppe (south, with Artemisia L. sp.), coast of the Black and Azov seas. General distribution: Southeast (south of Ukraine, Crimea, Russia: Rostov region) Europe; Southwest (Turkey, Iran) Asia.

5. L. syvaschicum Kleopov, 1939, Bot. Journ. Inst. Bot. Akad. Nauk URSR, 21-22: 251; Kotov, 1979, Fl. evrop. ch. USSR, 4: 60; Vasconcellos, Akeroyd, Rich, 1993, Fl. Europ. 1: 401.

Described from the Syvash Area (Ukraine). Lectotypus: "Lepidium crassifolium Waldst. et Kit. Po dnu vysohshoi Syvashskoi satoki na pivden vid s. Sakharivki Novotroitzkogo r-nu. 26.06.1931, O. Svistunova (По дну висохшої Сивашської затоки на південь від с.
Захарівки Новотроїцького р-ну. 26.06 .1931 р., О. Свистунова)" (KW) [Iljinska, 2002, Ukr. Botan. Journ. 59, 3: 261].

Distribution in Ukraine: Steppe (south), coast of the Black and Syvash seas. General distribution: Southeast Europe (Syvash Area), endemic.

6. L. latifolium L. 1753, Sp. P1.: 644; Kotov, 1979, Fl. evrop. ch. USSR, 4: 60; Szafer, Kulczyński, Pawłowski, 1986, Rośl. Polsk. 1: 233; Vasconcellos, Akeroyd, Rich, 1993, Fl. Europ. 1: 401; Ilyinska et al. 2007, Ecofl. Ukr. 5: 80 - L. latifolium subsp. eulatifolium Thell. 1906, Neue Denkschr. Schweiz. Ges. Naturw. 41: 159. - Cardaria latifolia (L.) Spach, 1838, Hist. Nat. Vég. (Spach), 6: 546. - Lepia latifolia (L.) Desv. 1814, Journ. Bot. Appl. (Paris), 3: 166.

Described from Europe: "Habitat in Galliae, Angliae umbrosis, succulentis". Lectotypus: Herb. Linn. No. 824.11a (LINN) [Jafri, 1973, in Nasir et Ali (ed.), Fl. W. Pakistan, 55: 60].

Distribution in Ukraine: rare in Zakarpattja, Carpathians, Roztochchja, Opillja and Polissja; common to Forest-steppe, Steppe and Crimea. General distribution: Europe (adventives into North, Center and West); Asia (adventives into East); Northern Africa; adventives into North America, South America and Australia.

7. L. turczaninowii Lipsky, 1894, Zap. Kiev. Obshch. Isteshvoispyt. (Mem. Soc. Nat. Kiev) 13: 412; Kotov, 1979, Fl. evrop. ch. SSSR, 4: 61; - L. lyratum L. subsp. lacerum auct. non (C. A. Mey.) Thell.: Vasconcellos, Akeroyd, Rich, 1993, Fl. Europ. 1: 401, p. p. - L. lyratum L. subsp. lacerum (C.A. Mey.) Thell. var. turczaninowii (Lipsky) Thell. 1906, Gatt. Lepid.: $166,169$.

Described from Crimea: "Tauria meridionali ad Theodosiam, in declivibus ad mare prope castellum vetus". Holotypus: "Lepidium turczaninowii sp. n. Крым, Феодосия. 21.07.1891 г., Липский” (LE !, in herb. pro typus).

Distribution in Ukraine: East Crimea. General distribution: Europe (Crimea), endemic.

8. L. campestre (L.) W. T. Aiton, 1812, Hortus Kew. ed. 2, 4: 88; Kotov, 1979, Fl. evrop. ch. SSSR, 4: 58; Vasconcellos, Akeroyd, Rich, 1993, Fl. Europ. 1: 399. - Thlaspi campestre L. 1753, Sp. Pl.: 646. - Lepia campestris (L.) Desv. 1815, J. Bot. Agric. 3: 165. Lasioptera campestris (L.) Andrz. ex DC. 1821, Syst. Nat. 2: 535, nom. nudum. - Iberis campestris (L.) Wallr. 1822, Sched. Crit.: 341.

Described from Europe "Habitat in Europee arvis, viis argillosis". Lectotypus: Herb. Linn. No 825.8 (LINN) [Hedge, 1965, Fl. Turkey, 1: 280]. 
Distribution in Ukraine: Zakarpattja, Carpathians, Roztochchja, Opillja, Pravoberezhne Polissja (rare), Forest-steppe, Steppe (rare), Mountain of Crimea (common); extends on the north. General distribution: Europe; Southwest (M. Asia, Caucasus) and East. (China) Asia; adventives into North America, South America, South Africa and Australia.

9. L. sativum L. 1753, Sp. Pl.: 644; Kotov, 1979, Fl. evrop. ch. SSSR, 4: 58; Vasconcellos, Akeroyd, Rich, 1993, Fl. Europ. 1: 399; Ilyinska et al. 2007, Ecofl. Ukr. 5: 79. - Lepia sativa (L.) Desv. 1814, Journ. Bot. Appl. (Paris), 3: 165.

Described from the cultivated plants of unknown origin: "Habitat - - _.". Lectotypus: Herb. Linn. No. 824.11 (LINN) [Fawcett et Rendle, 1914, in Fl. Yamaica, 3: 243].

Distribution in Ukraine: all territory; cultivated, as also escaped. General distribution: Europe; Southwest Asia; North America, South America; North Africa; Australia; native to Northeast Africa et Southwest Asia, in other regions cultivated, as also escaped.

10. L. perfoliatum L. 1753, Sp. Pl.: 643; Kotov, 1979, Fl. evrop. ch. SSSR, 4: 59; Szafer, Kulczyński, Pawłowski, 1986, Rośl. Polsk. 1: 232; Vasconcellos, Akeroyd, Rich, 1993, Fl. Europ. 1: 401. - Nasturtium perfoliatum (L.) Besser, 1822, Enum.: 26, N. 743. Crucifera diversifolia E.H.L. Krause, 1902, Deutschl. Fl. (Sturm), ed. 2, 6: 156.

Described from Southwest Asia: "Habitat in Persia, Syria". Lectotypus: Herb. Clifford: 331, "Lepidium 3" (BM-000646274) [Jonsell et Jarvis, 2002, Nordic J. Bot. 22: 70].

Distribution in Ukraine: Zakarpattja and Polissja (adventives?), Forest-steppe, Steppe, Crimea. General distribution: Europe (native to South and Southeast, adventives into all regions); Asia (adventives into East); North Africa; adventives into North America, South America and Australia.

11. L. ruderale L. 1753, Sp. P1.: 645; Kotov, 1979, Fl. evrop. ch. SSSR, 4: 59; Szafer, Kulczyński, Pawłowski, 1986, Rośl. Polsk. 1: 233; Vasconcellos, Akeroyd, Rich, 1993, Fl. Europ. 1: 401.- L. ambiguum Lange, 1865, in Vidensk. Meddel. Dansk Naturhist. Foren. Kjobenhavn, 7: 7 (non F. Müller, 1855, in Trans. Philos. Soc. Victoria, 1: 34). - Nasturtium ruderale (L.) Scop. 1772, Fl. Carn. 2, 2: 13. - Iberis ruderalis (L.) Crantz, 1762, Stirp. Austr. Fasc. 1: 21. - Thlaspi ruderale (L.) All. 1785, Fl. Pedem. 1: 250.

Described from Europe: "Habitat in Europee ruderatis et ad vias". Lectotypus: Herb. Linn. No. 824.17 (LINN) [Jonsell et Jarvis, 2002, Nordic J. Bot. 22: 70].
Distribution in Ukraine: all territory. General distribution: almost all Europe (but adventives into the North); Southwest (M. Asia, Caucasian and Caspian regions, Transcaucasia) Asia; adventives into North America and Australia.

12. L. pinnatifidum Ledeb. 1842, Fl. Ross. 1: 206; Kotov, 1979, Fl. evrop. ch. SSSR, 4: 59; Vasconcellos, Akeroyd, Rich, 1993, Fl. Europ. 1: 401; Ilyinska et al. 2007, Ecofl. Ukr. 5: 80.

Described from the Southeast Europe (Astrakhan area): "In regione astrachanensi! (Blume)". Lectotypus: "Inter plantas astrachanensi..., Blume D. 19" (LE) [Dorofeev, 2008, Botan. Journ. 93, 12: 1969].

Distribution in Ukraine: Steppe (one locality). General distribution: East Europe; Southwest (Afghanistan) and Central Asia; adventives into South America and North America.

Note. A single specimen of this species was collected by S. Korzhinsky in Melitopol (S.E. Ukraine) in 1880, and was determined by Latowski in 1977 (LE!). Today it is the only locality.

13. L. virginicum L. 1753, Sp. P1.: 645; Szafer, Kulczyński, Pawłowski, 1986, Rośl. Polsk. 1: 234; Vasconcellos, 1964, Fl. Europ. 1: 332; Kuusk, 1973, Eesti NSV Fl. 5: 535, tab. 286; Kotov, 1979, Fl. evrop. ch. SSSR, 4: 58; Vasconcellos, Akeroyd, Rich, 1993, Fl. Europ. 1: 401.

Described from North et Central America: "Habitat in Virginia, Jamaicae glareosis". Lectotypus: Herb. Linn. No. 824.18 (LINN) [Marais, 1970, in Cogg et al. (ed.), Fl. Southern Africa, 13: 94].

Distribution in Ukraine: adventives into Zakarpattja and Crimea. General distribution: native to North America; adventives into all regions: Europe, Asia, South America, South Africa, Australia.

14. L. densiflorum Schrad. 1832, Index Sem. Horti Götting.: 4; Kotov, 1979, Fl. evrop. ch. SSSR, 4: 59; Vasconcellos, Akeroyd, Rich, 1993, Fl. Europ. 1: 401; Ilyinska et al. 2007, Ecofl. Ukr. 5: 79. - L. apetalum auct. non Willd.: N. Busch, 1939, Fl. SSSR, 8: 508. - L. micranthum Ledeb. 1842, Fl. Ross. 1: 205. - L. divaricatum auct. non W.T. Aiton: Kuusk et al. 1993, in Fl. Balt. Republ. 1: 335.

Described from Göttingen Botanical Garden: "Venit in hortis sub L. praecoci et bipinnatifido, utraque specie a nostra diversissima". Holotypus: "Germany. Sine locus, 1831, Schrader s.n.” (Z).

Distribution in Ukraine: Zakarpattja, Carpathians, Roztochchja, Opillja, Polissja, Forest-steppe (rare), Steppe (rare). General distribution: native to North America, adventives into Europe, Asia and South America. 
Acknowledgments. I thank Dr. Vira Hayova (M. G. Kholodny Institute of Botany, Kiev) and Dr. Andriy V. Yena (Crimean
Agrotechnological University, Simferopol) for reading the English text.

\section{References}

Bowman J., Brüggemann H., Lee J.-Y. \& Mummenhoff K. 1999. Evolutionary changes in floral architecture within the genus Lepidium (Brassicaceae). Int. J. Plant Sci. 160(50): 917-929.

De Carvalho \& E Vasconcellos J. 1964. Lepidium L. In: T. G. Tutin, V. H. Heywood, N. A. Burges, D. H. Valentine, S. M. Waters \& D. A. Webb (eds.). Flora Europaea, vol. 1: 330-333. University Press, Cambridge.

Dorofeev V. I. 2012. Brassicaceae Burnett. In: D. V. GeltMAN (ed.) Conspectus florae Europae Orientalis, vol. 1: 364-437. Consociatio editionum scientificarum KMK, Petropoli-Mosqua.

Hewson H. J. 1982. The genus Lepidium L. (Brassicaceae) in Australia. Brunonia 4(2): 217-308.

ILYINSKA A. 2002. Typification of vascular plants described from the territory of Ukraine: family Brassicaceae (genera Erysimum L. - Turrutis L.). Ukr. Botan. Journ. 59(3): 259-267.

ILYINSKA A. P. 2005. The new data on distribution and systematic of some species Brassicaceae of the florae of Ukraine. Ukr. Botan. Journ. 62(3): 375-382.

ILYINSKA A. P. 2009. New data on distribution of some species of Brassicaceae in the western areas of Ukraine. Ukr. Botan. Journ. 66(3): 340-344.

Ilyinska A., Didukh Ya., Burda R., Korotchenko I. 2007. Ecoflora of Ukraine. Vol. 5. 584 pp. Phytosociocentre, Kiev.

Ilyinska A. P., Shevera M. V. 2004. The leaf surface of representatives of the genera Lepidium L., and Cardaria Desv. (Brassicaceae). Ukr. Botan. Journ. 61(2): 115124

Kotov M. I. 1953. Fam. Cruciferae Juss. In: M. V. KLOKov \& O. D. VisYulina (eds.). Flora of URSR, vol. 5, pp. 203-429. Academy of Sciences of URSR, Kiev.

Kotov M. I. 1979. Fam. Brassicaceae Burnett. In: An. A. Theodorov (ed.). Flora partis Europaeae URSS, vol. 4, pp. 30-148. Nauka, Leningrad.
Kotov M. I. 1987. Fam. Brassicaceae Burnett. In: J. M. ProKudin (ed.). Opredelitel' vysshikh rastenǐ̌ Ukrainy, pp. 109-129. M. G. Kholodny Institute of Botany, National Academy of Sciences of Ukraine, Kiev.

LAtowski K. 1982. Taksonomiczne studium karpologiczne eurazjatyckich gatunków rodzaju Lepidium L. Wyd. Nauk. UAM, seria Biologia, 23, 105 pp. Poznań.

Lee J-Y., Mummenhoff K. \& Bowman J. L. 2002. Allopolyploidization and evolution of species with reduced floral structures in Lepidium L. (Brassicaceae). Proceedings of the National Academy of Sciences, USA. 99(26): 16835-16840.

Mummenhoff K., Bowman J. L., Linder H. P., Friesen N. \& FranZKe A. 2004. Molecular evidence for bicontinental hybridogenous genomic constitution in Lepidium sensu stricto (Brassicaceae) species from Australia and New Zealand. Am. J. Bot. 91(2): 252-259.

Mummenhoff K., Brüggemann H. \& Bowman J. L. 2001. Chloroplast DNA phylogeny and biogeography of Lepidium (Brassicaceae). Am. J. Bot. 88(11): 2051-2063.

Mummenhoff K., Polster A., Mühlhausen A. \& Theissen G. 2009. Lepidium as a model system for studying the evolution of fruit development in Brassicaceae. J. Exp. Bot. 60(5): 1503-1513.

Protopopova V. V., Mosyakin S. L. \& Shevera M. V. 1994. Lepidium virginicum L. - new species in Ukrainian flora. Ukr. Botan. Journ. 51(2-3): 225-228.

Smirnov P. A. 1948. About Lepidium Meyeri Claus. Byulleten Moskovskogo obschestva ispytatelej prirody. Otdel Biologicheskij. 53(4): 72-78.

Thellung A. 1906. Die Gattung Lepidium (L.) R. Br. Eine monographische Studie. Neue Denkschr. Allg. Schweiz. Ges. Naturwiss. 41(1): 1-340.

Wastowicz P. \& Rostański A. 2009. The use of quantitative characters in determination of frequently misdiagnosed species within Lepidium L. sect. Dileptium (Brassicaceae). Acta Soc. Bot. Pol. 78(3): 221-2217. 